\title{
Performance of Sampling/Resampling-based Particle Filters Applied to Non-Linear Problems
}

\author{
Nga Ly-Tu${ }^{1}$, Thuong Le-Tien ${ }^{2}$, Oanh Tran-Hoang-Thi ${ }^{3}$, Tu Huynh-Kha ${ }^{1}$, Linh Mai ${ }^{4}$ \\ ${ }^{1}$ Computer Science and Engineering, International University, Ho Chi Minh City, Vietnam \\ 2 Electrical and Electronics Engineering, Ho Chi Minh City University of Technology, Vietnam \\ ${ }^{3}$ Electrical and Engineering, Ho Chi Minh City University of Technical Education, Vietnam \\ 4 Electrical and Electronics Engineering, International University, Ho Chi Minh City, Vietnam
}

Correspondence: Nga Ly-Tu, ltnga@hcmiu.edu.vn

Manuscript communication: received 18 December 2014, accepted 4 May 2015

\begin{abstract}
In this work, we propose a wireless body area sensor network (WBASN) to monitor patient position. Localization and tracking are enhanced by improving the effect of the received signal strength (RSS) variation. First, we propose a modified particle filter (PF) that adjusts resampling parameters for the Kullback-Leibler distance (KLD)-resampling algorithm to ameliorate the effect of RSS variation by generating a sample set near the high-likelihood region. The key issue of this method is to use a resampling parameter lower bound for reducing both the root mean square error (RMSE) and the mean number of particles used. To determine this lower bound, an optimal algorithm is proposed based on the maximum RMSE between the proposed algorithm and the KLD-resampling algorithm or based on the maximum mean number of particles used of these algorithms. Finally, PFs based on KLD-sampling and KLD-resampling are proposed to minimize the efficient number of particles and to reduce the estimation error compared to traditional algorithms.
\end{abstract}

Keywords- KLD-resampling, KLD-sampling, non-linear problems, SIR.

\section{INTRODUCTION}

The current challenge in wireless body area sensor network (WBASN) is to estimate physical parameters associated to the patient such as the location in space, the moving velocity, etc. This is a difficult problem due to its non-linear characteristics $[1,2]$. There exist several approaches to deal with this problem. The most recent approach is recursive Bayesian filtering that uses a set of particles with assigned primary weights serves as the basic idea of a particle filter (PF) [2]. Bayesian filters have been applied to solve location estimation problems [3]. Bayesian filters are also a kind of statistics in which all available information is utilized to reduce the number of uncertainty present in decision-making. New information is incorporated in foregoing information to create the basis for statistical procedures. The formal mechanism that combines new information with previously available one is known as the Bayes theorem.

Over the past decade, most research in wireless communications has emphasized the use of PFs for solving many problems. Core PFs are introduced in [4] to determine communication problems, such as blind equalization, blind detection over flat fading channels, multiuser detection, and estimation/detection of spacetime codes in fading channels. An approximation technique called unscented PF $[5,6]$ is employed to reduce degeneration of the particle in wireless sensor networks via received signal strength (RSS) measurement, which is a particular type of functional auxiliary sampling density design. In [7], a new method for evaluation of the number of particles in a wireless sensor network model was derived.

Recently, a number of authors have considered the effects of choosing metric and weight functional approach on PFs. In [8], the PF based on the KullbackLeibler distance (KLD)-sampling algorithm is proposed in order to determine the minimum number of particles needed to maintain the approximation quality in the sampling process. Then, in [9], KLD-sampling with adjusted sampling parameter, which adjusts standard deviation and then uses gradient data, is proposed to further improve the operation time and the sample set size for tracking the position of mobile robots in three-dimensional vectors by generating samples which are near the high-likelihood region. In contrast to the above KLD-sampling algorithms, the KLD-resampling algorithm proposed in $[10,11]$ determines the number of particles to resample so that the KLD between the distributions of particles before and after resampling does not exceed a pre-specified error bound.

In [12], we introduced an enhanced PF for the KLDresampling algorithm by adding an adjusted resampling parameter so as to create a sample set near the high-likelihood region. By setting up a specific lower bound for the resampling parameter, we found that this technique further reduces the estimation error of target but maintains the proper KLD-resampling [11] for WBASNs because the variation of RSS measurement values is the diminished. 
From the brief review of the literature above, it can be observed that while the KLD-sampling algorithm in [9] does not employ a lower bound on the sampling paramemter, the KLD-resampling algorithm in [12] did consider the lower bound of the resampling parameters but that bound was only based on experiments. Moreover, the lower bound of the resampling parameter in [12] is only a special case of Remark 1 in Section 3.

The main contributions of this paper are summarized as follows. First, for the underlying system in [12], namely System 1 in this paper, we extend it by proposing an algorithm to obtain the lower bound values of the resampling parameter based on two criteria. The first criterion (Remark 1) is to maximize the gap between the RMSEs of the proposed algorithm and the KLD-resampling algorithm. The second criterion (Remark 2) is to maximize the gap between the mean numbers of particles of these algorithms. These remarks are given in Algorithm 6.

Second, for the typical non-linear system given in [13], namely System 2 in this paper, we show that with 50 particles the sampling importance resampling (SIR) algorithm based on PF implemented on MATLAB and FPGA hardware are nearly the same. Given the strong contribution of the selected metric and weight functional methods, we use them in the proposed model for assessing the number of used particles based on MATLAB before testing on board.

The paper is organized as follows. Introduction to the systems of interest is given in Section 2. All related schemes, namely, SIR, KLD-sampling, KLD-resampling, and our method are presented in Section 3. The RMSE criterion and all experimental results based on MAT$\mathrm{LAB}$ for tracking the position of the target of the nonlinear problem and WBASN are shown in Section 4. Finally, we conclude the paper in Section 5.

\section{SYSTEMS}

Non-linear problems are generally characterized by a discrete-time state-space model at time $k$ as follows [1]:

$$
\begin{aligned}
& x_{k}=g_{k}\left(x_{k-1}, u_{k-1}, w_{k-1}\right), \\
& z_{k}=h_{k}\left(x_{k}, v_{k}\right),
\end{aligned}
$$

where $x_{k}$ are unobserved system states, $z_{k}$ are observed signal measurements, $u_{k}$ is the control system input, $w_{k}$ and $v_{k}$ are respectively process and observation noise given in terms of their the probability distribution functions (pdf), $g_{k}(\cdot)$ and $h_{k}(\cdot)$ are the state transition and observations functions and they are generally nonlinear.

A large number of applied signal processing problems are formulated as a special case of (1) and (2), in which the noise processes are assumed to enter additively according to the given formulation. We here consider all systems under the benchmark model of maneuvering target tracking in a two-dimensional plane to evaluate the sample size adjusting ability of the KLDresampling algorithm. Then, the system state vector in (1) is given in more detail as

$$
x_{k}=T_{1}\left(A x_{k-1}+B u_{k}\right)+T_{2} C w_{k}
$$

where, given the notation that $x_{k}=\left[x_{1, k}, x_{2, k}, x_{3, k}, x_{4, k}\right]^{T}$, $\left(x_{1, k}, x_{3, k}\right)$ and $\left(x_{2, k}, x_{4, k}\right)$ are the position coordinates of the target and its velocities along the $x$ and $y$ axes, $u_{k}$ and $w_{k}$ are the control input and process noise vectors, $A, B$ and $C$ are some constants, and

$$
T_{1}=\left[\begin{array}{llll}
1 & T & 0 & 0 \\
0 & 1 & 0 & 0 \\
0 & 0 & 1 & T \\
0 & 0 & 0 & 1
\end{array}\right], \quad T_{2}=\left[\begin{array}{cc}
\frac{T^{2}}{2} & 0 \\
T & 0 \\
0 & \frac{T^{2}}{2} \\
0 & T
\end{array}\right],
$$

with $T$ being the sampling period.

In addition, the bearing-only measurement for an observer at the origin, that is (2), can be reformulated based on the following systems.

\subsection{System 1}

In recent years, a number of studies have considered the issue of tracking patient position in WBASN [1416]. A WBASN consists of a collection of various wireless networked low-power biosensor devices, which integrate an embedded microprocessor, radio and a limited amount of storage. These known positions can be either measured during the deployment or estimated by the WBASN itself using a cooperative localization algorithm [17, 18].

In our paper, we only solved the problem of indoor localization based on PF in WBASN. The state system model for the wireless biomedical sensor localization [5] is investigated and reformulated for evaluating the required number of particles as

$$
\begin{aligned}
& x_{k}=T_{1}\left(x_{k-1}+V_{k} \Delta t\right)+T_{2} w_{k}, \\
& z_{k}=P_{\text {ref }}+K \log \left(\arctan \left(x_{1, k} / x_{3, k}\right)\right)+v_{k},
\end{aligned}
$$

where $x_{k}$ is the position of a mobile node from the starting point, $z_{k}$ is the RSS measurement, $P_{\text {ref }}$ is the reference value of RSS, $K$ is the factor in path loss, $V_{k}$ is velocity of mobile node and is uniformly distributed over the pseudo random range of $\left[V_{\min }, V_{\max }\right]$ over a time segment $\triangle t, w_{k}$ and $v_{k}$ are the process and measurement noise with distributions of $\mathcal{N}(0, Q)$ and $\mathcal{N}(0, R)$ respectively, and the sampling period $T$ expressed in $T_{1}$ and $T_{2}$ is equal to 1 .

\subsection{System 2}

Similar to System 1, our previous system [13] is currently reconsidered and reformulated [10] for assessing sample size based on KLD-resampling/sampling as follows:

$$
\begin{aligned}
& x_{k}=T_{1}\left(0.917 x_{k-1}+0.25 u_{k}\right)+T_{2} w_{k}, \\
& z_{k}=0.334 \arctan \left(x_{1, k} / x_{3, k}\right)+v_{k},
\end{aligned}
$$

where $u_{k}$ is a uniformly distributed pseudo random number, the process noise covariance matrix, $Q$, and the measurement noise variance, $R$, might change over time and measurement but we assume here that they are constant, and the sampling period $T=1$. 


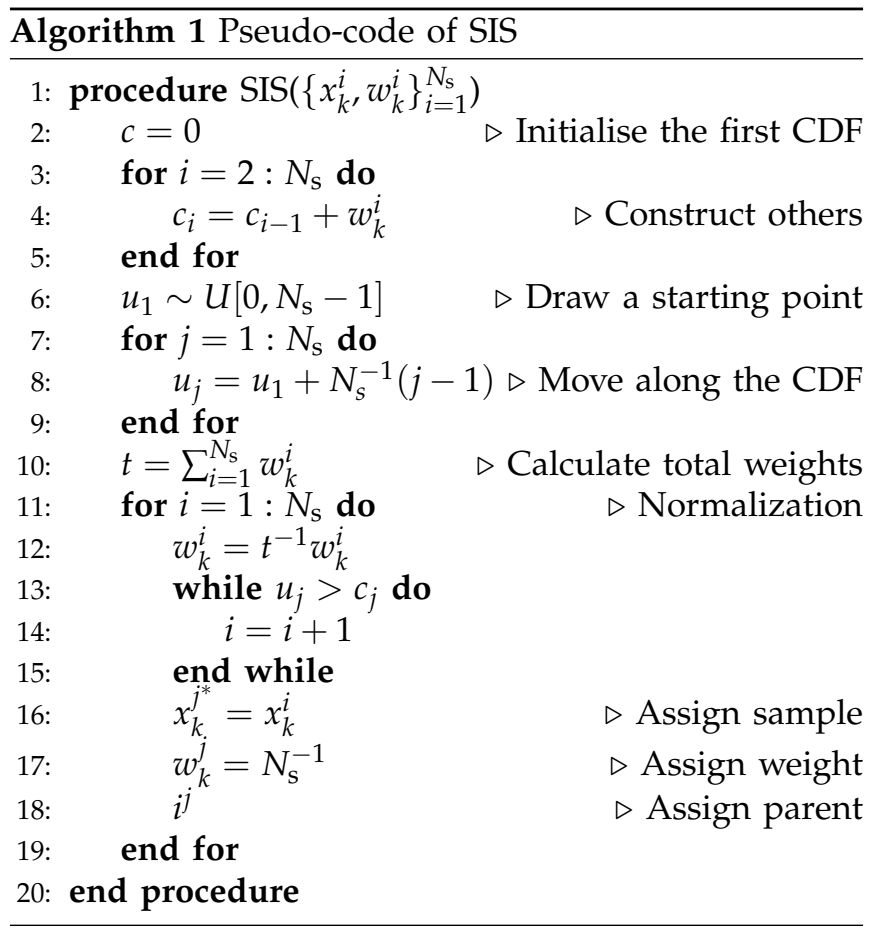

\section{Particle Filters}

In this section, we first review the PFs based on the SIR, KLD-sampling, and KLD-resampling algorithms. Then, we present the proposed algorithm, which is the KLDresampling with adjusted resampling parameter based on $\mathrm{PF}$, in Algorithm 5. Finally, the solution to these optimal resampling parameter lower bound values is shown in Algorithm 6.

\subsection{SIR}

The concept of auxiliary PF has been introduced in $[2,4-7,13,19-23]$. A PF is also known as a bootstrap filter, Monte Carlo technique, condensation algorithm, interacting particle approximations and survival of the fittest. The key idea is to represent the required posterior density function by a set of random samples (particles) with associated primary weights, and to compute the estimates based on the samples and primary weights. As the number of samples becomes very large, the Monte Carlo characterization is the closest equivalent representation of the posterior probability function, and the solution approaches the optimal Bayesian. The sequential importance sampling (SIS) algorithm [21], shown in Algorithm 1 for the PF, includes a resampling step at each instant as described in detail in the reference. The SIS algorithm uses the important density, which is a proposed density to represent another one that cannot be exactly computed, that is the sought posterior density in the present case. Hence, samples are drawn from the important density instead of the actual density. The degeneracy phenomenon is known as a common problem with the SIS PF, which all but one particle has negligible primary weight after a few states. Thus, a large computational effort is devoted to updating particles whose contribution to the approximation of the posterior density function is almost zero.

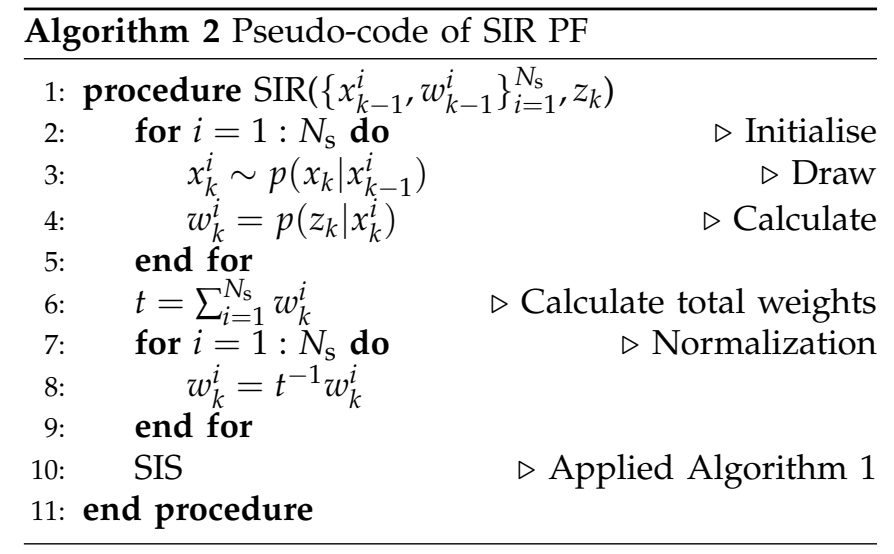

This problem can be overcome by increasing the number of particles, or more efficiently by approximately selecting the important density. In addition, the use of the resampling technique in [21] is recommended to avoid the degeneracy of the particles, as Algorithm 1. The pseudo-code of SIR PF is shown in Algorithm 2.

\subsection{KLD-sampling}

This subsection briefly discusses the KLD-sampling method in [8]. In the sampling process, as these individuals in the population are sorted by non-domination, the use of a fast KLD-sampling technique, called an adaptive $\mathrm{PF}$ at each iteration of the PF, determines the number of samples such that with probability $1-\delta$ the error between the true posterior and the sample-based approximation is less than $\epsilon$.

KLD is used to show how to determine the number of samples so that the distance between the sample-based maximum likelihood estimate and the true posterior does not exceed a pre-specified threshold $\epsilon$.

The KLD between the proposal distribution, $q$, and the true distribution, $p$, is defined in discrete form as

$$
d_{\mathrm{KL}} \triangleq \sum_{x} p(x) \log \frac{p(x)}{q(x)}=\sum_{x} W(x) q(x) \log W(x),
$$

where $W(x)=p(x) / q(x)$. The required number of

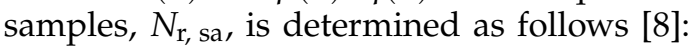

$$
N_{\mathrm{r}, \mathrm{sa}}=\frac{1}{2 \epsilon} \chi_{k-1,1-\delta}^{2}
$$

where $k$ is the number of bins with support, and the quantiles of the Chi-square distribution are computed as

$$
P\left(\chi_{k-1}^{2} \leq \chi_{k-1,1-\delta}^{2}\right)=1-\delta .
$$

Based on the Wilson-Hilferty transformation [24], an approximation of $\chi_{k-1,1-\delta}^{2}$ in (9) is given by

$$
N_{\mathrm{r}, \mathrm{sa}}=\frac{k-1}{2 \epsilon}\left[1-\frac{2}{9(k-1)}+\sqrt{\frac{2}{9(k-1)}} z_{1-\delta}\right]^{3},
$$

where $z_{1-\delta}$ is the upper $1-\delta$ quantile of the standard normal distribution.

\subsection{KLD-resampling}

The undesirable results above are caused by the number of particles needed to approximate a discrete 


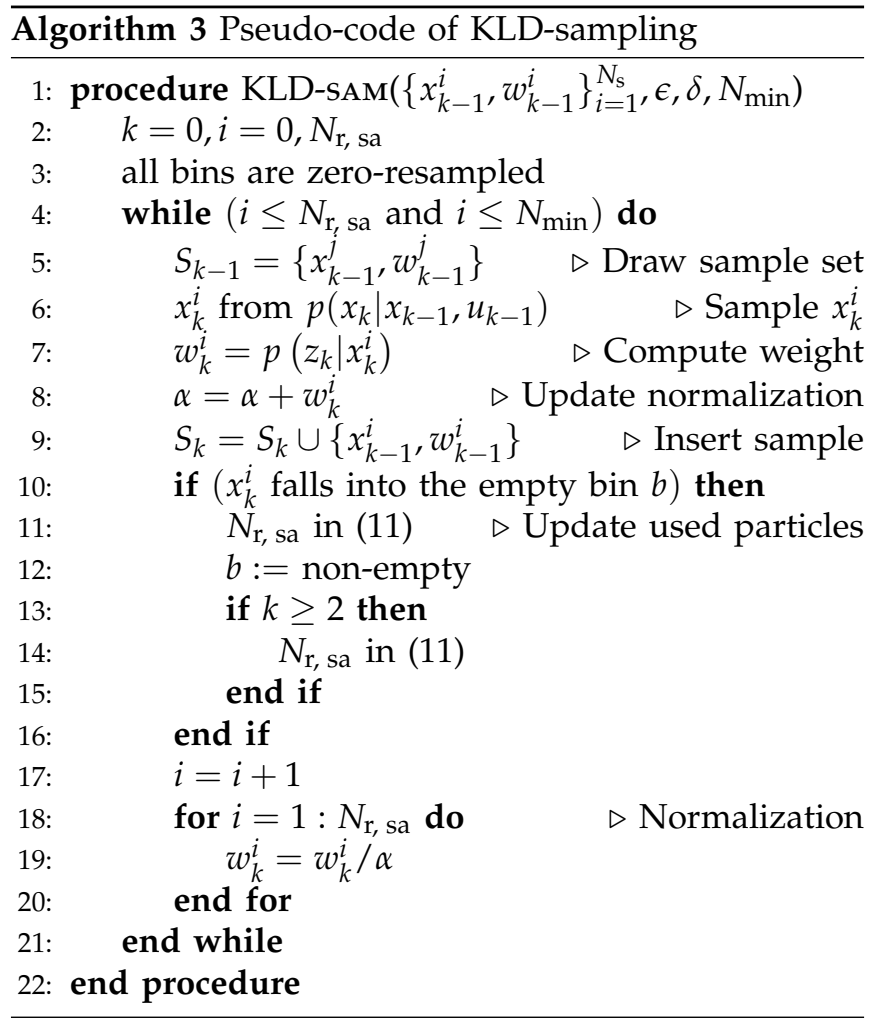

distribution with an upper bound $\epsilon$ on the KLD. In other words, the KLD-sampling method leads to statistical bounds of the approximation quality of samples that are actually drawn from the proposal distribution rather than the true posterior. The mismatch between the true posterior and the proposal distributions is ignored. To avoid this mismatch, the result in (11) is applied in the resampling process to determine the total number of particles to resample. The authors in [10] proposed to divide the particles of the posterior distribution into bins and count the number of bins, $k$, in which at least one particle is resampled to determine the total number of particles to resample. This method is called KLD-resampling. Therefore, the required number $N_{\mathrm{r}, \mathrm{sa}}$ in (11) is replaced by

$$
N_{\mathrm{r}, \mathrm{re}}=\min \left\{N_{\max }, \operatorname{ceil}\left(N_{\mathrm{r}, \mathrm{sa}}\right)\right\},
$$

where $N_{\mathrm{r} \text {, sa }}$ is defined in (11).

\subsection{Proposed Algorithm}

In this subsection, we discuss two problems. First, we describe our KLD-resampling with adjusted resampling parameter algorithm for applied PF. Second, we present a way to determine the optimal resampling parameter lower bound values for our method, as shown in Algorithm 6.

3.4.1 KLD-Resampling with Adjusted Resampling Parameter: The authors in [9] proposed that KLDsampling with the help of an adjusted sampling parameter improves RMSE values. This sampling parameter is adjusted by increasing the standard deviation, which is inversely proportional to the likelihood, and generating samples near the true distribution or near the highlikelihood region. By the similarity to this method,

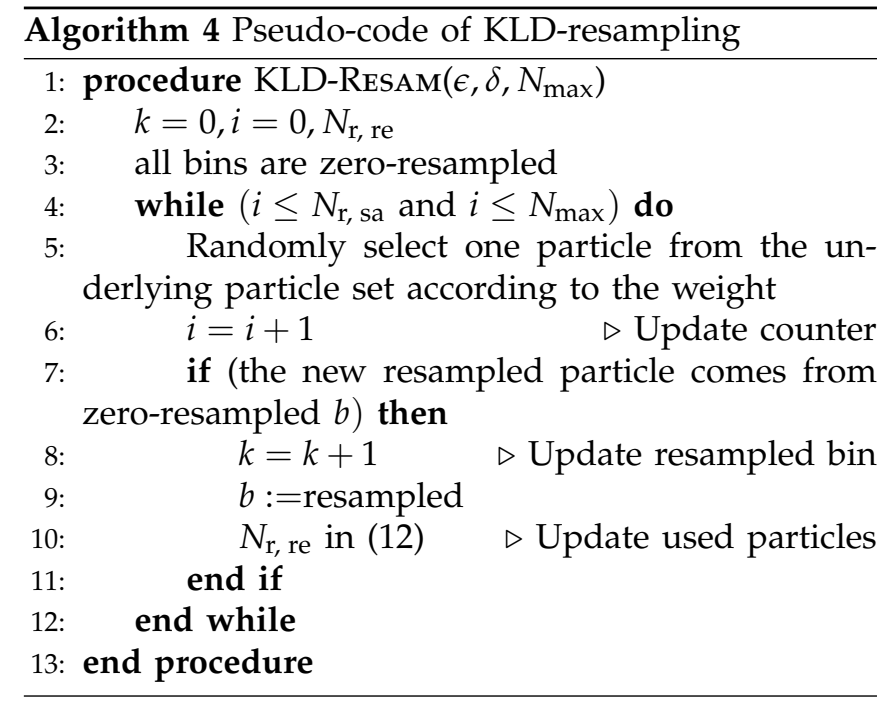

we propose a method that incorporates the KLDresampling with an adjusted resampling paramter. First, the adjusted standard deviation is computed using the relationship between the maximum number of samples and the number of required samples as

$$
\sigma_{\mathrm{ad}}=\sigma_{\mathrm{lb}}+\epsilon \frac{N_{\mathrm{r}, \mathrm{re}}}{N_{\max }},
$$

where $\sigma_{\mathrm{ad}}$ and $\sigma_{\mathrm{lb}}$ are respectively the adjusted standard deviation and the resampling parameter lower bound. Next, the new samples are drawn using the following rule:

$$
x_{k}^{i+N_{\mathrm{r}, \mathrm{re}}}= \begin{cases}x_{k}^{i}+\sigma_{\mathrm{ad}} \mathrm{randn}, & \text { if }\left.(\exists i) \frac{\partial p(h(x))}{\partial x}\right|_{x=x_{k}^{i}} \\ x_{k}^{i}-\sigma_{\mathrm{ad}} \text { randn, } & \text { otherwise, }\end{cases}
$$

where

$$
\left.\frac{\partial p(h(x))}{\partial x}\right|_{x=x_{k}^{i}}=\left.\frac{\partial}{\partial x}\left[\frac{1}{\sigma \sqrt{2 \pi}} \exp \left\{-\frac{\left(z_{k}-h(x)\right)^{2}}{2 \sigma^{2}}\right\}\right]\right|_{x=x_{k}^{i}}
$$

is the gradient at the state, and $\sigma$ is the variance of a Gaussian pdf of the measurement model. Then, the new samples are used to update the weights in order to generate the new samples in the high-likelihood region. As a result, the operation time is reduced and the tracking accuracy is increased because of the small sample set size. Our algorithm is summarized in Algorithm 5.

3.4.2 Resampling Parameter Lower Bound: We propose an algorithm to determine the optimal resampling parameter lower bound $\sigma_{\mathrm{lb} \text {, opt }}$, as shown in Algorithm 6 . Let us denote $\sigma_{\mathrm{lb}, i}$ to be the resampling parameter lower bound of the $i$ th $\mathrm{RMSE}_{\sigma_{\mathrm{lb}, i},}^{\mathrm{Pro}}$ which is the RMSE value of the proposed algorithm, $\sigma_{\mathrm{lb}, i}, \mathrm{RMSE}^{\mathrm{KLD}}$ and RMSE $^{\text {SIR }}$ are respectively the RMSE values of KLDresampling and SIR, $\left(N_{\mathrm{r}, \text { re }}\right)_{\sigma_{\mathrm{lb}, i}}^{\mathrm{Pro}}$ and $N_{\mathrm{r}, \mathrm{KLD}}$ are the mean numbers of particles used of the proposed algorithm and the KLD-resampling algorithm, respectively, and $\sigma_{\mathrm{lb}}^{* 1}$ (line 14) and $\sigma_{\mathrm{lb}}^{* 2}$ (line 19) are respectively the sets of resampling parameter lower bound that fulfil the condition of Remark 1 (line 13) and Remark 2 (line 18).

Let us define

$$
\triangle \mathrm{RMSE}=\left[\triangle \mathrm{RMSE}_{\sigma_{\mathrm{lb}, 1}^{* 1}}, \ldots, \triangle \mathrm{RMSE}_{\sigma_{l b, L}^{* 1}}\right],
$$




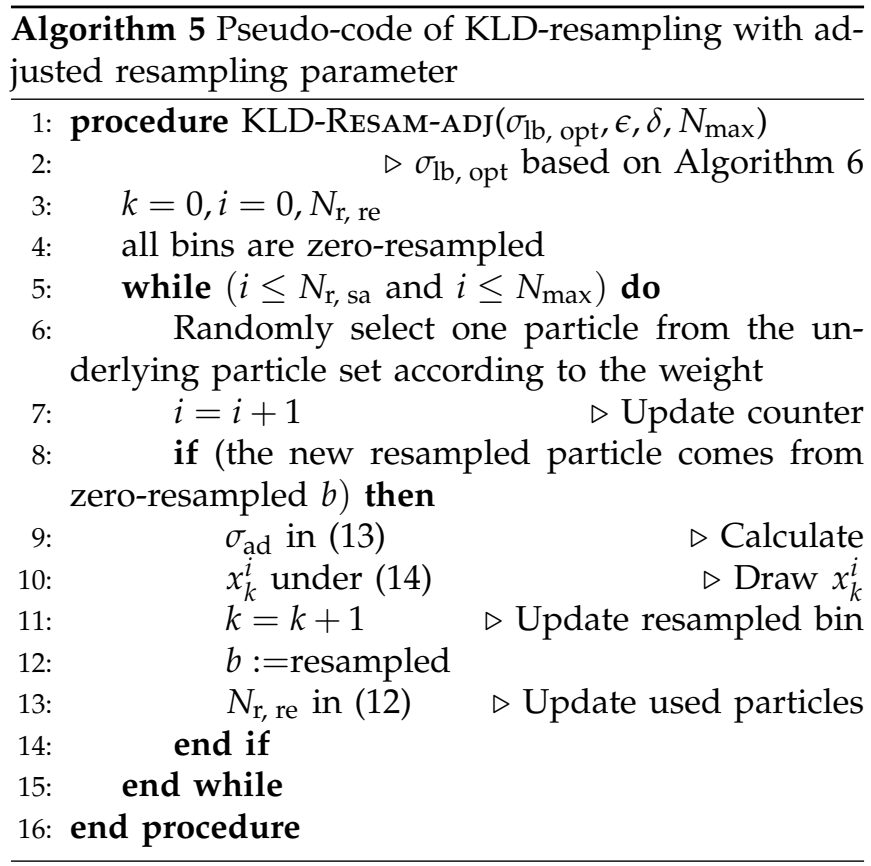

where

$$
\triangle \mathrm{RMSE}_{\sigma_{l b, i}^{* 1}}=\mathrm{RMSE}_{\sigma_{\mathrm{lb}, i}^{* 1}}^{\mathrm{Pro}}-\mathrm{RMSE}^{\mathrm{KLD}}
$$

is the gap of RMSE value between proposed algorithm and the KLD-resampling algorithm, and

$$
\triangle N=\left[\triangle N_{\sigma_{\mathrm{lb}, 1}^{* 2}}, \ldots, \triangle N_{\sigma_{l b, Q}^{* 2}}\right]
$$

where

$$
\triangle N_{\sigma_{\mathrm{bb}, i}^{* 2}}=\left(N_{\mathrm{r}, \mathrm{re}}\right)_{\sigma_{\mathrm{lb}, i}^{* 2}}^{\mathrm{Pro}}-N_{\mathrm{r}, \mathrm{KLD}}
$$

is the gap mean number of particles between the proposed algorithm and the KLD-resampling algorithm.

Algorithm 6 provides the following two optimal lower bound values for the resampling parameter:

1) Remark 1: if $\triangle \mathrm{RMSE}_{\sigma_{\mathrm{lb}, i}}>0$ (lines 13 to 17 ), then a value $\sigma_{\mathrm{lb} \text {, opt }}^{* 1}($ line 25$)$ exists to maximize the function $\triangle$ RMSE.

2) Remark 2: if $\Delta N_{\sigma_{\mathrm{lb}, i}}>0$ (lines 18 to 21), then a value $\sigma_{\mathrm{lb}}^{* 2}$, opt $($ line 26) also exists to maximize the function $\triangle N$.

\section{Simulation Results}

In this section, we first define the RMSE criterion. Next, we conduct a series of simulations to compare the mean number of particles used, RMSE, and operation time of SIR, KLD-sampling, KLD-resampling, and our method for the two systems. All simulations are run on PC Core i5-2400 @ 3.10 GHz, 4.00 GB RAM and MATLAB 2012a (7.14.0.739). We assume that process and measurement noises are Gaussian for reasons of convenience.

\subsection{RMSE}

The authors in [10] showed the inconsistency of the physical location of the given points to estimate location. A lower RMSE indicates high accuracy of the

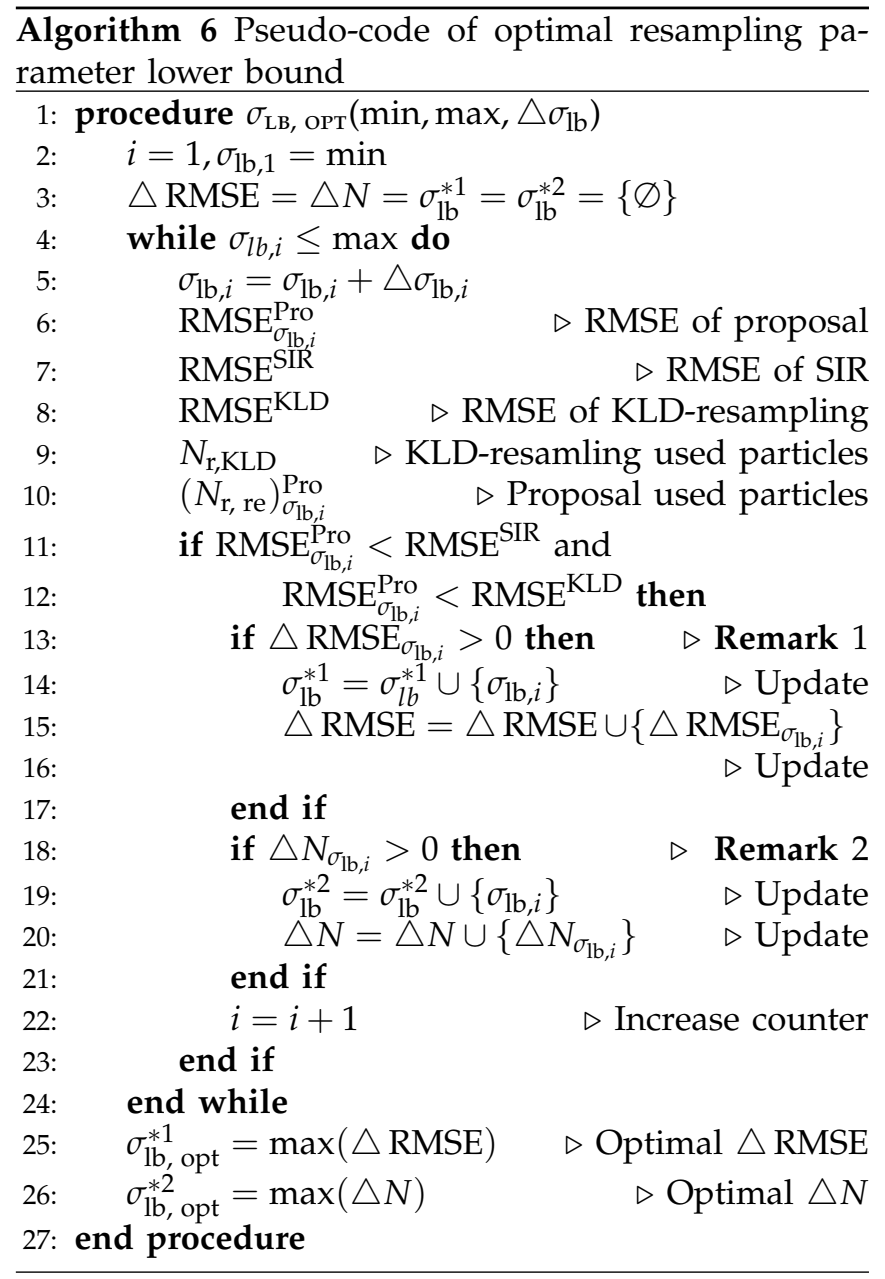

transformation estimated. To determine the RMSE, the residuals are considered the difference between the actual and predicted values. Therefore, in case of a two-dimensional plane, the RMSE is used to determine the Euler distance between the distance and the true position of the target, and is defined as

$$
\text { RMSE }=\sqrt{\left(x_{1, k}-\hat{x}_{1, k}\right)^{2}+\left(x_{3, k}-\hat{x}_{3, k}\right)^{2}},
$$

where $\hat{x}_{1, k}$ and $\hat{x}_{3, k}$ are the estimated $x$ and $y$ positions of the target at certain time.

\subsection{System 1}

We conducted a series of simulations to determine the adjusted variance in (13) based on Algorithm 6. Next, the performance of position tracking, RMSE, and the mean number of particles used for all methods are evaluated considering the two remarks.

The $\sigma_{\mathrm{lb}}$ under Algorithm 6 is considered in Table I. The parameters are assumed and simulated as follows [10]: bound parameters $\epsilon=0.25, \delta=0.01$, the bin size is smaller than the standard deviations of the dynamic model and the measurement $(Q=I$, i.e. identity matrix, and $R=1), N_{\mathrm{s}}=400, N_{\max }=N_{\mathrm{s}} / 2, V_{\max }=5$, $V_{\min }=1, V_{\text {init }}=5, K=-45, P_{\text {ref }}=-23$, and the length of time is 40 for sample size variation in one trial (iteration) with various values of $\sigma_{\mathrm{lb}}$ from 0.5 to 4 . The set of indices $\sigma_{\mathrm{lb}}^{* 1}=[0.25,0.5,1,1.25,1.5,1.75,2,3,3.25,3.5]$ 
Table I

RMSE vs. Mean Number of Particles Used

\begin{tabular}{|c|c|c|c|c|c|c|c|}
\hline \multirow{2}{*}{$\sigma_{\mathrm{lb}}$} & \multicolumn{3}{|c|}{ RMSE } & \multicolumn{2}{c|}{ Mean number of particles used } & \multirow{2}{*}{$\triangle$ RMSE } & $\triangle N$ \\
\cline { 2 - 6 } & SIR & KLD-resampling & Proposal & KLD-resampling & Proposal & & \\
\hline 0.25 & 0.3497 & 0.4822 & 0.2137 & 9.325 & 8.675 & 0.2685 & 0.650 \\
\hline 0.50 & 0.3473 & 0.2953 & 0.2855 & 9.875 & 9.250 & 0.0098 & 0.625 \\
\hline 0.75 & 0.4471 & 0.6778 & 0.6068 & 9.550 & 7.975 & - & - \\
\hline 1.00 & 0.2031 & 0.3483 & 0.1211 & 8.425 & 8.475 & 0.2272 & - \\
\hline 1.25 & 0.7291 & 0.4353 & 0.2447 & 9.800 & 7.300 & 0.1906 & 2.500 \\
\hline 1.50 & 0.3712 & 0.5384 & 0.2417 & 8.950 & 9.125 & 0.2967 & - \\
\hline 1.75 & 0.3196 & 0.4429 & 0.2844 & 9.200 & 8.725 & 0.1585 & 0.475 \\
\hline 2.00 & 0.3256 & 0.2835 & 0.1641 & 10.05 & 9.825 & 0.1194 & 0.225 \\
\hline 2.25 & 0.1607 & 0.2637 & 0.2738 & 9.850 & 9.625 & - & - \\
\hline 2.50 & 0.1813 & 0.4211 & 0.2170 & 8.650 & 10.325 & - & - \\
\hline 2.75 & 0.1683 & 0.5723 & 0.1816 & 9.451 & 9.075 & - & - \\
\hline 3.00 & 0.1501 & 0.2346 & 0.2082 & 9.152 & 9.150 & - & - \\
\hline 3.25 & 0.1718 & 0.1160 & 0.1098 & 9.575 & 9.700 & 0.0062 & - \\
\hline 3.50 & 0.6135 & 0.3351 & 0.1677 & 9.375 & 9.125 & 0.1674 & 0.250 \\
\hline 3.75 & 0.1160 & 0.3158 & 0.748 & 9.302 & 9.350 & - & - \\
\hline 4.00 & 0.2831 & 0.2525 & 0.5305 & 9.350 & 9.725 & - & - \\
\hline
\end{tabular}

fulfils $\triangle \mathrm{RMSE}_{\sigma_{\mathrm{lb}, i}}>0$. Based on Remark $1, \sigma_{\mathrm{lb}, \mathrm{opt}}^{* 1}=1.5$ because $\triangle \mathrm{RMSE}_{\sigma_{l b, o p t}^{* 1}}=0.2967$. The set of indices $\sigma_{\mathrm{lb}}^{* 2}=$ $[0.25,0.5,1.25,1.75,2,3.5]$ fulfils $\Delta N_{\sigma_{\mathrm{bb}, i}}>0$. Based on Remark 2, $\sigma_{\mathrm{lb} \text {, opt }}^{* 2}=1.25$ because $\Delta N_{\sigma_{\mathrm{lb}}^{*} \text { opt }}=2.5$. The results in Table I shows the estimation error (RMSE criterion) and the mean number of particles used of technique at $\sigma_{\mathrm{lb} \text {, opt }}^{* 1}=1.5$ and $\sigma_{\mathrm{lb} \text {, opt }}^{* 2}=1.25$, which are lower than those of the other methods. Thus, we set up this value for our proposed method in the next section, and simulations were conducted to compare the RMSE criterion, the number of particles used, and tracking target for all approaches.

4.2.1 Performance of Remark 1: Figures 1 to 3 show the performance of all methods under Remark 1 in terms of the mean tracking position, the RMSE, and the mean number of particles used. Clearly, as shown in Figure 1, the tracking of the target using the proposed algorithm is more accurate than that using the others. The RMSE curves for the three algorithms are shown in Figure 2, which verifies that the RMSE value of the proposed algorithm is lower than that of the others. For instance, from time 20 to 40 , the gap between the proposed algorithm and KLD-resampling regularly increases from about 13 to around 34, respectively. From time 8 to 13, the curve of the proposed algorithm is slightly higher than the curve of KLD-resampling by about 1 to 5 . Before discussing the performance of Remark 2, a comparison of the mean number of particles used for the proposed algorithm and KLDresampling is given in Figure 3. The overall number of particles used in the proposed algorithm is almost equal to the number of particles used in KLD-resampling.
4.2.2 Performance of Remark 2: In the same way, the mean tracking position, the RMSE, and the mean number of particles used for all algorithms were compared under Remark 2, as shown in Figures 4 to 6. Figure 4 shows that the trajectory estimated by the proposed algorithm is nearest to the true trajectory when the target is moving from 100 to around 600. In Figure 5, the RMSE curve of our algorithm always lies lower than that of the others for the whole time. More importantly, Figure 6 confirms that the mean number of particles used in the proposed algorithm is lower than that used in KLD-resampling by about 2.5 .

\subsection{System 2}

The observation of System 2 is a first-order function and thus its derivation in (14) has a positive value. As such, KLD-resampling with adjusted resampling parameter is not considered here. As shown by the implementation results [13], our system works well in reality with the number of particles being 50 . Thus, this system is considered in cases wherein the adapting sample size of particles is less than 50 . The parameters of Figures 7 to 9 are set as follows: length of time $k=40$, $N_{\mathrm{s}}=100, N_{\min }=50, N_{\max }=50, Q=0.01 I, R=0.01$, $\epsilon=0.65, \delta=0.01$ and sample size variation in 10 trials.

Figures 7 to 9 show the comparison of the mean numbers of used particles and of the RMSE, both for the three SIR, KLD-sampling, and KLD-resampling PFs. Similar to KLD-sampling, the mean number of particles used in KLD-resampling to obtain the maximum of 65 at time 1 , which sharply reduces at time 4 , and reaches the lowest near 11 from 5 to 40, as shown in Figure 7 . The mean RMSE of KLD-sampling achieves around 


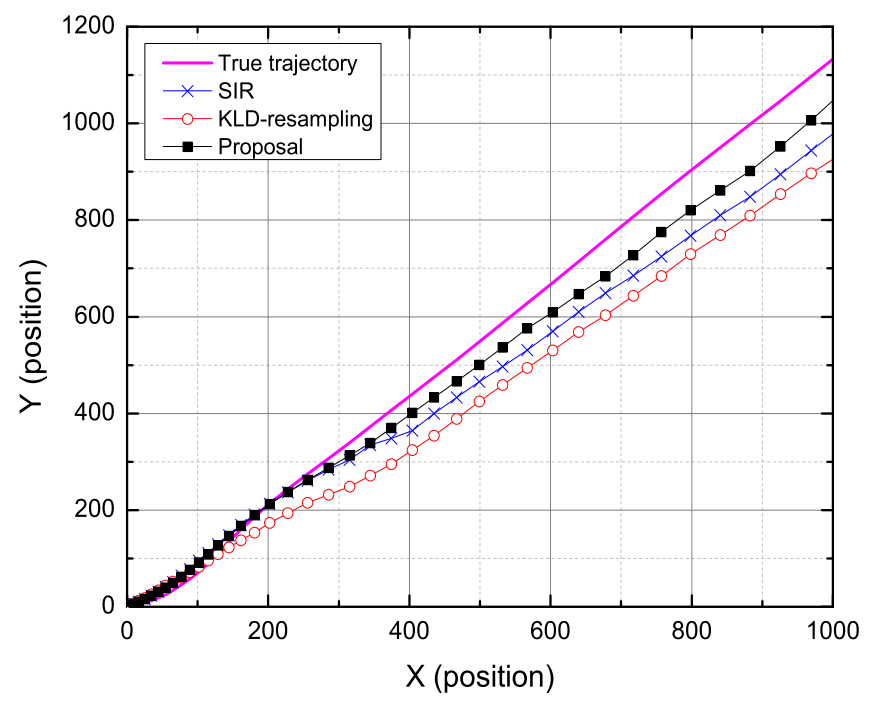

Figure 1. Mean tracking position of System 1 under Remark 1.

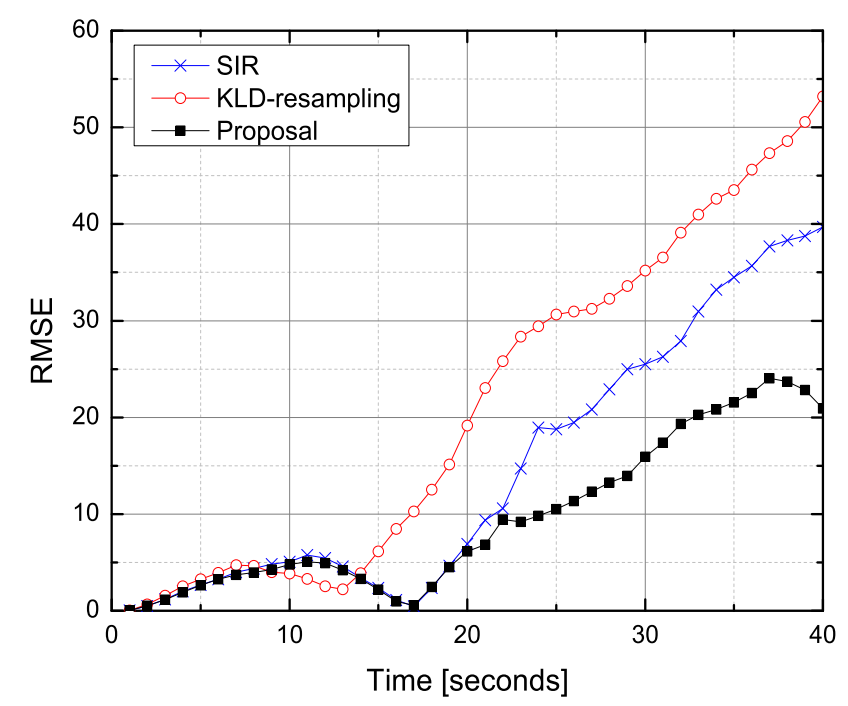

Figure 2. RMSE of System 1 under Remark 1.

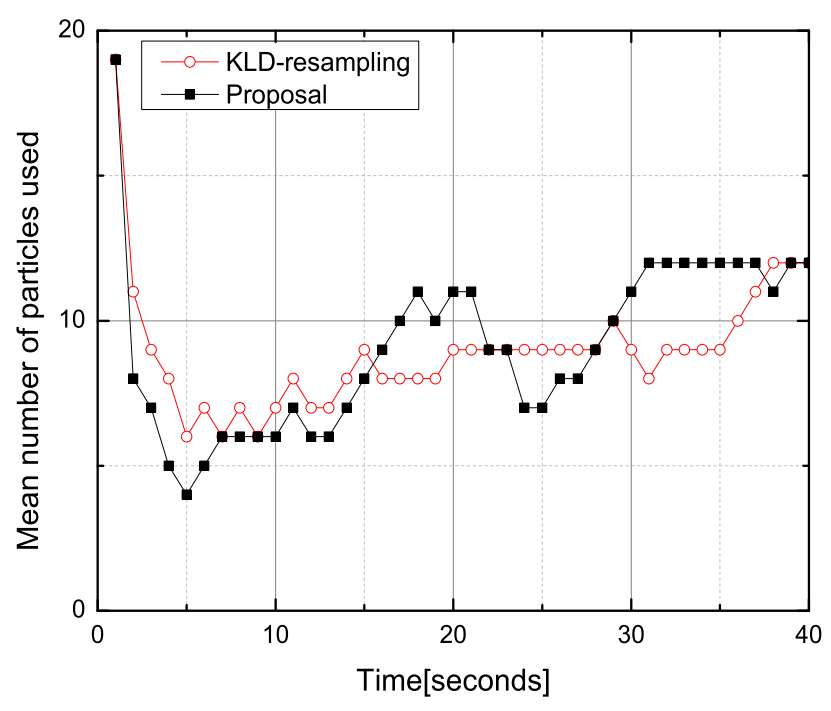

Figure 3. Mean number of particles used of System 1 under Remark 1.

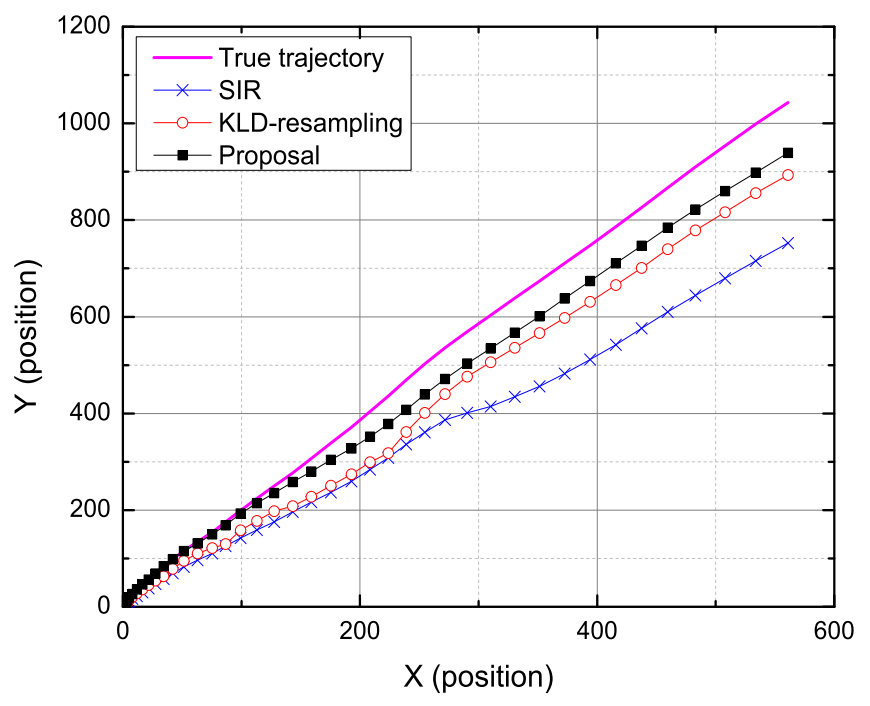

Figure 4. Mean tracking position of System 1 under Remark 2.

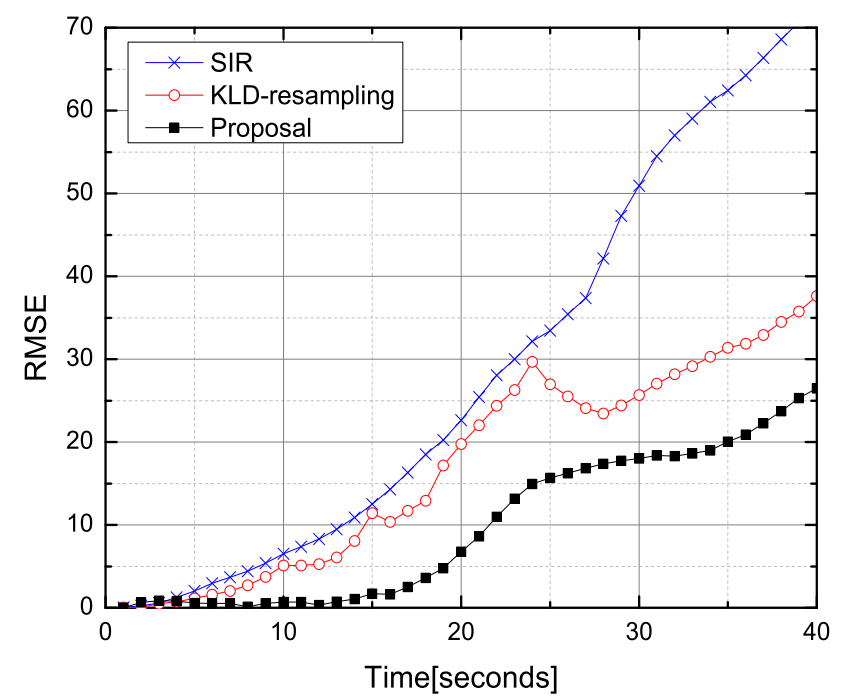

Figure 5. RMSE of System 1 under Remark 2.

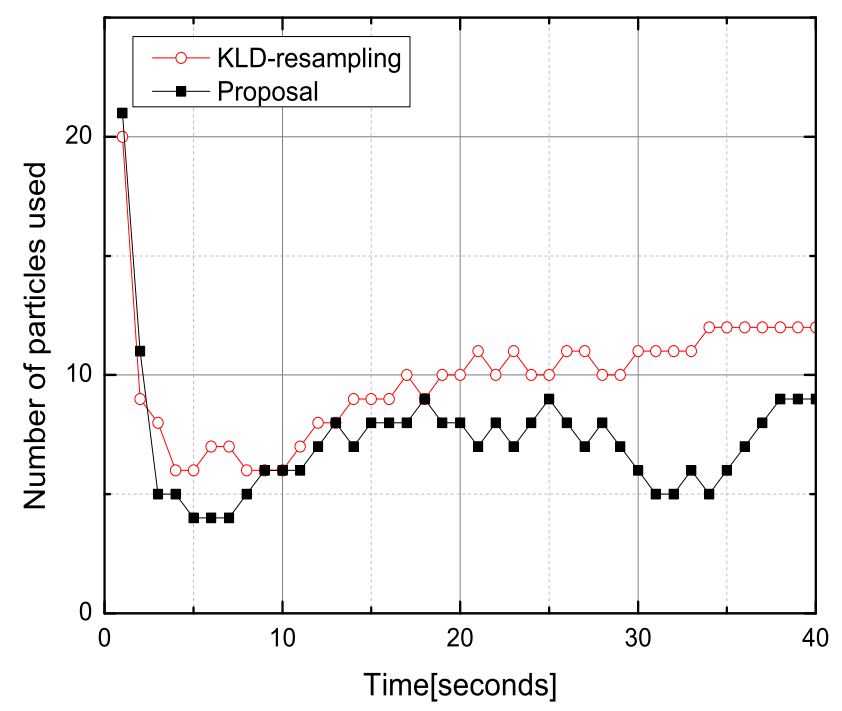

Figure 6. Mean number of particles used of System 1 under Remark 2. 


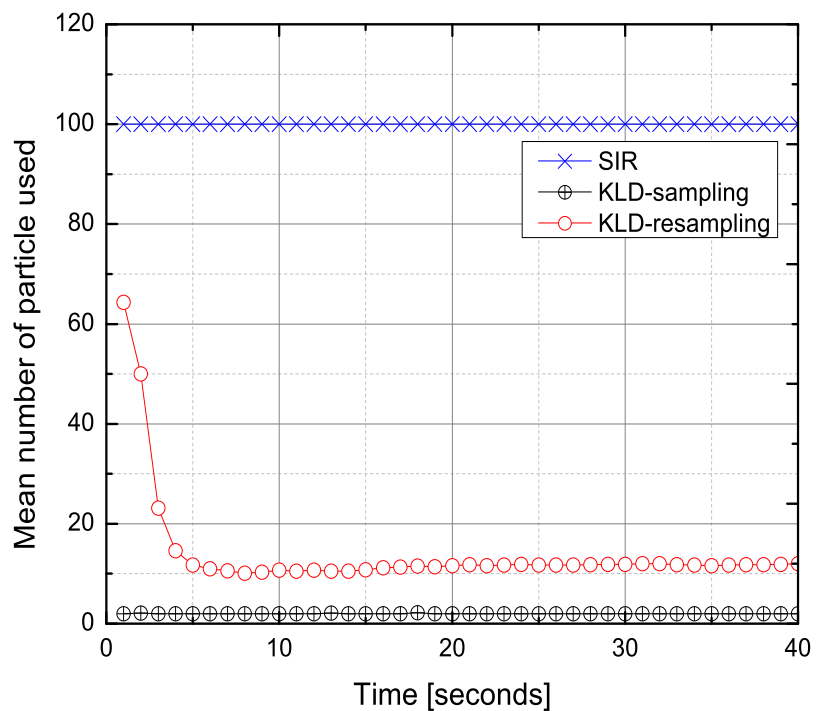

Figure 7. Mean number of particles used of System 2.

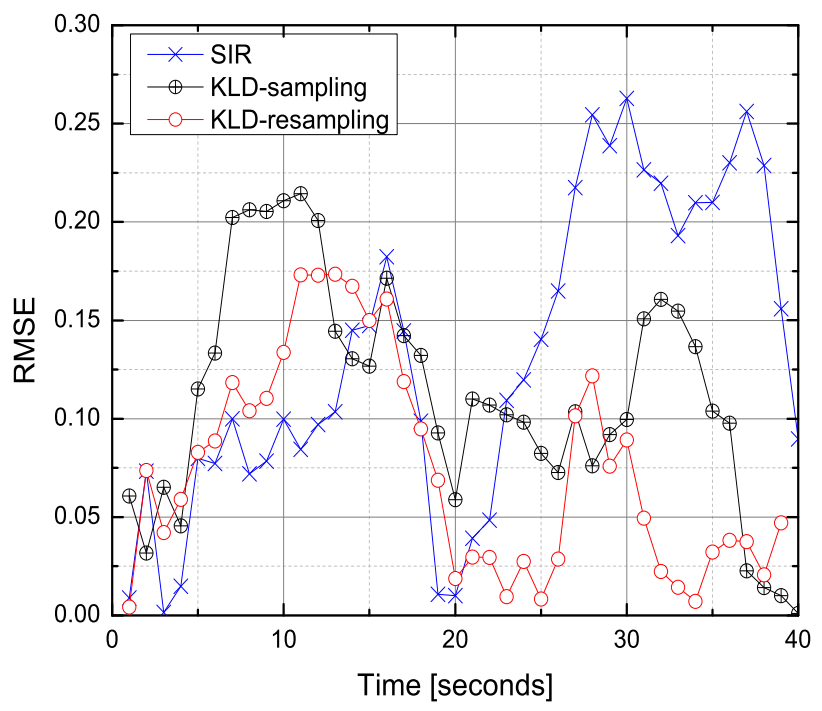

Figure 8. RMSE of System 2.

0.1121, and of KLD-resampling around 0.0741 and both are lower than that of SIR, as shown in Figure 8. In addition, the mean number of the required particles for KLD-sampling is fixed, i.e., few particles (about two particles) with operation time that is similar to that of KLD-resampling (row 2, Table II below) and slightly higher than that of SIR. Finally, the traditional PF is not beneficial with respect to reduction of the sample size. Moreover, the mean tracking position using the three algorithms is shown in Figure 9. The target tracking using KLD-resampling and KLD-sampling is more accurate than that using SIR. The SIR curve slightly moves away from the true target with the $x$ position changing from 10 to around 18 .

The PF uses the trial process to eliminate the noise in the system and the measurement. Actually, a higher number of trials provides more accurate results in a generic system state. Table II presents the RMSE and the operation time with various numbers of trials

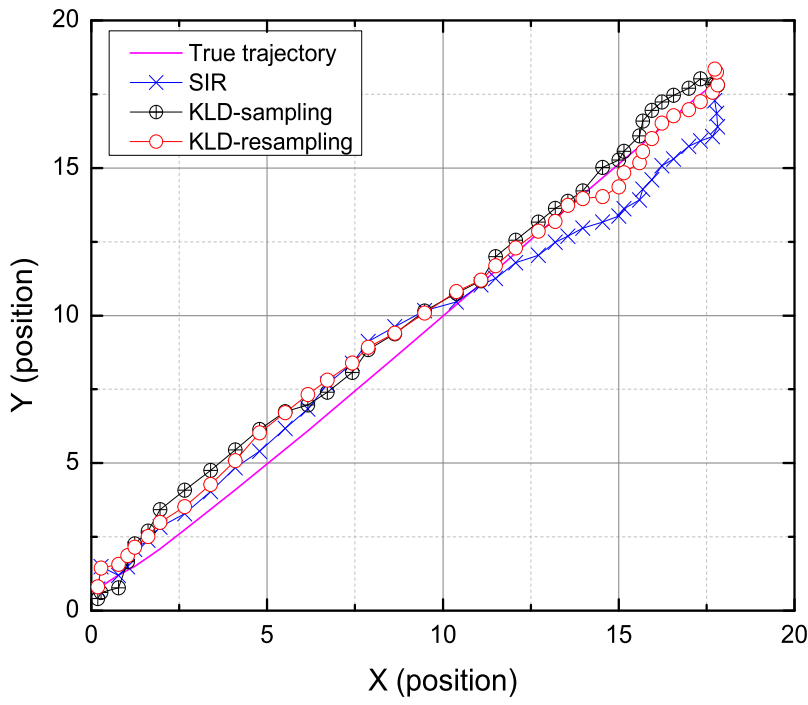

Figure 9. Mean tracking position of System 2.

Table II

RMSE AND OPERATION TIME WITH VARIOUS NUMBER OF TRIALS FOR THE THREE ALGORITHMS IN SYSTEM 2

\begin{tabular}{|c|c|c|c|}
\hline \multirow{2}{*}{$\begin{array}{c}\text { No. } \\
\text { Trials }\end{array}$} & \multicolumn{3}{|c|}{ (RMSE; operation time) } \\
\cline { 2 - 4 } & SIR & KLD-resampling & Proposal \\
\hline 1 & $0.0192 ; 0.0135$ & $0.0195 ; 0.0197$ & $0.0161 ; 0.0175$ \\
\hline 10 & $0.0054 ; 0.0607$ & $0.0022 ; 0.0777$ & $0.0021 ; 0.0747$ \\
\hline 20 & $0.0025 ; 0.1189$ & $0.0022 ; 0.1412$ & $0.0020 ; 0.1421$ \\
\hline 30 & $0.0009 ; 0.1687$ & $0.0025 ; 0.2249$ & $0.0014 ; 0.2028$ \\
\hline 40 & $0.0013 ; 0.2173$ & $0.0014 ; 0.2818$ & $0.0011 ; 0.2733$ \\
\hline
\end{tabular}

for the evaluated algorithms. The estimation error of KLD-resampling and KLD-sampling decreases quickly when the number of trials increases regularly. The operation time of KLD-resampling is less than that of KLD-sampling in (11) because of the proper minimum number of required particles given in (12).

\section{Conclusion}

In this paper, a KLD resampling algorithm is proposed in order to adjust the resampling parameters for reducing the fluctuations of RSS samples. Ultimately, the patient localization problem is solved using a WBASN with a PF system structure that is designed by these obtained parameters. Furthermore, in using a resampling parameter lower bound, this approach reduces the number of used particles and provides more accurate patient localization. We conduct a large set of simulations to evaluate the sample size and the effect of different parameters via resampling parameter lower bound values. In addition, the number of used particles is reduced, and the KLD-resampling/sampling method is successfully applied for solving non-linear problems describing the systems of interest. For future work, we will continue to evaluate PFs based on an efficient KLD-resampling/sampling algorithm for our systems on board. 


\section{ACKNOWLEDGMENT}

This research was funded by Vietnam National University Ho Chi Minh City (VNU-HCM) under grant number C2015-28-03. The authors would like to thank the International University of the VNU-HCM for its support and the anonymous reviewers for their valuable comments.

\section{REFERENCES}

[1] A. Seppänen, M. Vauhkonen, P. J. Vauhkonen, A. Voutilainen, and J. P. Kaipio, "State estimation in process tomography- Three dimensional impedance imaging of moving fluids," International Journal for Numerical Methods in Engineering, vol. 73, no. 11, pp. 1651-1670, 2008.

[2] T. B. Schön, "Solving Nonlinear State Estimation Problems Using Particle Filters - An Engineering Perspective," Linköping University, Linköping, Tech. Rep. LiTHISY-R-2953, 2010.

[3] D. Fox, J. Hightower, L. Liao, D. Schulz, and G. Borriello, "Bayesian filtering for location estimation," IEEE pervasive computing, no. 3, pp. 24-33, 2003.

[4] P. M. Djurić, J. H. Kotecha, J. Zhang, Y. Huang, T. Ghirmai, M. F. Bugallo, and J. Miguez, "Particle filtering," IEEE Signal Processing Magazine, vol. 20, no. 5, pp. 19-38, 2003.

[5] Z. Wang, X. Zhao, and X. Qian, "Unscented particle filter with systematic resampling localization algorithm based on RSS for mobile wireless sensor networks," in Eighth International Conference on Mobile Ad-hoc and Sensor Networks (MSN). IEEE, 2012, pp. 169-176.

[6] R. V. D. Merwe, A. Doucet, N. D. Freitas, and E. Wan, "The unscented particle filter (Report No. TR 380)," Cambrige University, Tech. Rep., 2000.

[7] T. D. Nguyen, H. T. Trinh, V. D. Nguyen, N. T. Nguyen, T. T. Nguyen, and W. Koichiro, "A new evaluation of particle filter algorithm and apply it to the wireless sensor networks," in International Conference on Computing, Management and Telecommunications (ComManTel). IEEE, 2013, pp. 169-174.

[8] D. Fox, "Adapting the sample size in particle filters through KLD-sampling," The international Journal of robotics research, vol. 22, no. 12, pp. 985-1003, 2003.

[9] S.-H. Park, Y.-J. Kim, H.-C. Lee, and M.-T. Lim, "Improved adaptive particle filter using adjusted variance and gradient data," in IEEE International Conference on Multisensor Fusion and Integration for Intelligent Systems (MFI 2008). IEEE, 2008, pp. 650-655.

[10] T. Li, S. Sun, and T. P. Sattar, "Adapting sample size in particle filters through KLD-resampling," Electronics Letters, vol. 49, no. 12, pp. 740-742, 2013.

[11] T. Li, M. Bolic, and P. M. Djuric, "Resampling methods for particle filtering: classification, implementation, and strategies," Signal Processing Magazine, IEEE, vol. 32, no. 3, pp. 70-86, 2015.

[12] N. Ly-Tu, T. Le-Tien, V.-T.-L. Phuong, and M. Linh, "Particle filter through Kullback-Leibler distance resampling with adjusted variance and gradient data for wireless biomedical sensor networks," in Proceedings of the 9th International Conference on Ubiquitous Information Management and Communication. ACM, 2015, p. 4.

[13] T. Le-Tien, O. Tran-Thi-Hoang, G. Luong-Q, N. Ly-Tu, $\mathrm{M}$. On-Vu-N, and Duy-Nguyen-B, "Particle filter design for the system assumption implemented on Matlab and FPGA XILINX VIRTEX-II-Pro," in 17th International Conference on Analog VLSI (AVIC2014), Ho Chi Minh City, Vietnam, Oct. 2014, pp. 227-232.

[14] A. Redondi, M. Chirico, L. Borsani, M. Cesana, and M. Tagliasacchi, "An integrated system based on wireless sensor networks for patient monitoring, localization and tracking," Ad Hoc Networks, vol. 11, no. 1, pp. 39-53, 2013.

[15] A. Redondi, M. Tagliasacchi, M. Cesana, L. Borsani, P. Tarrío, and F. Salice, "LAURA-LocAlization and Ubiquitous monitoRing of pAtients for health care support," in IEEE 21st International Symposium on Personal, indoor and mobile radio communications workshops (PIMRC Workshops). IEEE, 2010, pp. 218-222.

[16] H. Ren, M. Q. Meng, and X. Chen, "Physiological information acquisition through wireless biomedical sensor networks," in IEEE International Conference on Information Acquisition. IEEE, 2005, pp. 483-488.

[17] N. Patwari, J. N. Ash, S. Kyperountas, A. O. Hero III, R. L. Moses, and N. S. Correal, "Locating the nodes: cooperative localization in wireless sensor networks," IEEE Signal Processing Magazine, vol. 22, no. 4, pp. 5469, 2005.

[18] M. Coates, "Distributed particle filters for sensor networks," in Proceedings of the 3rd international symposium on Information processing in sensor networks (IPSN). New York, USA: ACM, 2004, pp. 99-107.

[19] N. Gordon, B. Ristic, and S. Arulampalam, Beyond the Kalman filter: particle filters for tracking applications. Artech House, Boston, British Library, 2004.

[20] J. Carpenter, P. Clifford, and P. Fearnhead, "Improved particle filter for nonlinear problems," IEE ProceedingsRadar, Sonar and Navigation, vol. 146, no. 1, pp. 2-7, 1999.

[21] M. S. Arulampalam, S. Maskell, N. Gordon, and T. Clapp, "A tutorial on particle filters for online nonlinear/non-Gaussian Bayesian tracking," IEEE Transactions on Signal Processing, vol. 50, no. 2, pp. 174-188, 2002.

[22] A. Doucet, N. Freitas, and N. Gordon, Sequential Monte Carlo methods in practice. New York, Springer Publishers, 2001.

[23] B. D. Anderson and J. B. Moore, Optimal filtering. Prentice-Hall, New Jersey, America Dover Publications, 1999.

[24] N. L. Johnson, S. Kotz, and N. Balakrishnan, Continuous univariate distributions. New York, John Wiley and Sons Publishers, 1995. 


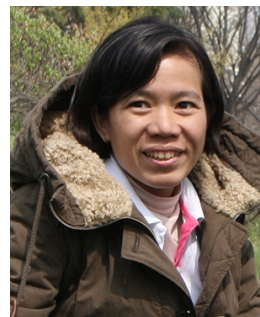

Nga Ly-Tu received her B.Eng. degree in Electronics and Telecommunications and M.Eng. degree in Radio Engineering and Electronics from the University of Technology, Ho Chi Minh City, Vietnam, in 1994 and 2004, respectively. From 2001 to 2004, she was a researcher at the Broadcasting Radio and Application Center in Vietnam. From 2004 to 2008, she worked as a Lecturer at the Industrial University, Vietnam. Since 2008, she has been working as a Researcher at the International University, Vietnam. Her research activities are in the area of embedded systems, heath care systems, and filters.

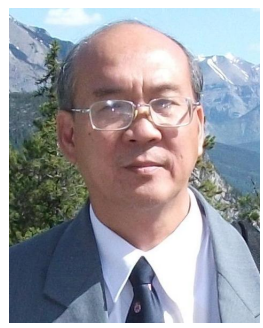

Thuong Le-Tien was born in Saigon, Ho Chi Minh City, Vietnam. He received the Bachelor and Master Degrees in EE-Engineering from Ho Chi Minh City University of Technology, Vietnam in 1980 and 1995 in respectively, then the Ph.D. in Telecommunications from the University of Tasmania, Australia, in 1998. Since May 1981 he has been a faculty member of the Telecommunications Department at Ho Chi Minh City University of Technology. He spent 3 years in the Federal Republic of Germany as a visiting scholar at the Ruhr University from 1989 until 1992. He served as Deputy Department Head for many years and had been the Telecommunications Department Head from 1998 until 2002. He had also appointed for the second position as the Director of Center for Overseas Studies since 1998 up to May 2010. His areas of specialization include: Communication Systems, Digital Signal Processing and Electronic Circuits. He has published more than 120 research articles and the teaching materials for university students related to Electronic Circuits 1 and 2, Digital Signal Processing and Wavelets, Antenna and Wave Propagation, Communication Systems. Professor Le-Tien was awarded the title as National Distinguished Lecturer and various certificates for his engineering education contributions from the Academic State Council and the Chairman of National. He has been a member of the IEEE since 1996.

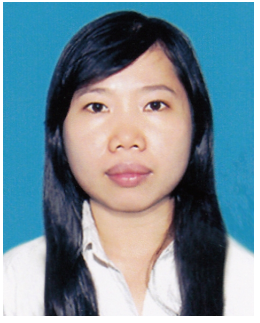

Oanh Tran-Thi-Hoang was born in 1982 and received her Bachelor degree and Master degree in Electrical and Electronic Technology from the Ho Chi Minh City University of Technical Education in 2012 and 2014, respectively. Her research interests include FPGA and design signal processing.

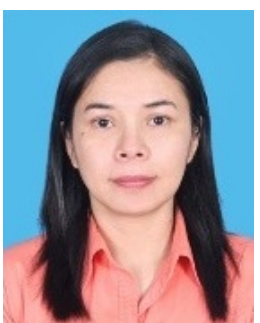

Tu Huynh-Kha received the Bachelor degree and Master degree in Electronics Engineering in 2001 and 2004 from Ho Chi Minh City University of Technology, Vietnam. At present, she is lecturer of the International University of Ho Chi Minh City and currently working toward the $\mathrm{PhD}$ degree in Electronics Engineering at Ho Chi Minh City University of Technology. Her research interests include signal processing, computer vision and image forensics.

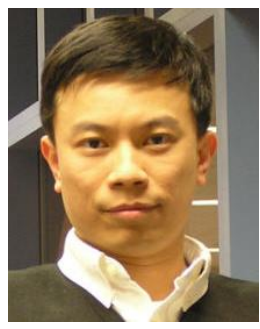

Linh Mai received The B.S. degree from Hanoi Natural Science University, in 1996, and M.S. Degree in ITIMS, Hanoi, Vietnam, in 1998, and Ph.D degree in Electrical Engineering from Korea Advanced Institute of Science and Technology, South Korea, in 2008. Since 2008 he has been with the School of Electrical Engineering, International University, Vietnam National University Ho Chi Minh city, Vietnam. His research interests include RF MEMS, RF IC Design, smart antenna, and MIMO. 